\title{
Thin AMC substrate by applying square patch with semicircle notch
}

\author{
Ryota Shinozaki ${ }^{1, \text { a)}}$, Jo Tamura ${ }^{1}$, and Hiroyuki Arai ${ }^{1}$ \\ ${ }^{1}$ Graduate School of Engineering, Yokohama National University, \\ 79-5 Tokiwadai, Hodogaya-ku, Yokohama, Kanagawa 246-8501, Japan \\ a)shinzoaki-ryota-xb@ynu.jp
}

Abstract: This paper presents a method of thinning an Artificial Magnetic Conductor (AMC) substrate by applying the semicircle notch to the square patch. We analyze the reflection characteristics by changing the arc length of the semicircle. As a result, the frequency where a Perfect Magnetic Conductor (PMC) characteristics appears is shifted to the lower side as the arc length of the semicircle increases. It is confirmed that the proposed method is thinning the AMC substrate thickness about 15\% compared with the AMC that has no semicircle notch. Furthermore, it was experimentally confirmed that the thin AMC substrate was thinning by semi-circle notch.

Keywords: AMC, PMC characteristics, thin antenna

Classification: Antennas and Propagation

\section{References}

[1] J. Li, et al., "Reflectarray element using interdigital gap loading structure," Electron. Lett., vol. 47, no. 2, pp. 83-85, Jan. 2011. DOI: 10.1049/el.2010.3083

[2] R. Dewan, S.K. Rahim, S.F. Ausordin, and T. Purnamirza, "The improvement of array antenna performance with the implementation of an artificial magnetic conductor (AMC) ground plane and in-phase superstrate," Progress in Electromagnetics Research Length, pp. 147-167, 2013. DOI: 10.2528/PIER13040206

[3] M. Abu, E.E. Hussin, R.F. Munawar, and H. Rahmalan, "Design synthesis of $5.8 \mathrm{GHz}$ octagonal AMC on a very thin substrate," International Journal of Information and Electronics Engineering, vol. 5, no. 5, pp. 376-380, 2015. DOI: 10.7763/IJIEE.2015.V5.562

[4] K.P. Gaurav, S.S. Hari, and K.M. Manoj, "Investigations of triple band artificial magnetic conductor back plane with UWB antenna," Microwave and Optical Technology Letters, vol. 58, no. 8, pp. 1900-1906, May 2016. DOI: 10.1002/ mop. 29943

[5] M. Abu, E.E. Hussin, and A.R. Othman, "Design of $0.92 \mathrm{GHz}$ artificial magnetic conductor for metal object detection in RFID tag application with little sensitivity to incidence of angle," Journal of Theoritical and Information Technology, vol. 60, no. 2, pp. 307-313, Feb. 2014.

[6] CST Microwave Suite, http://www.cst.com 


\section{Introduction}

In recent years, the development of the Internet of Things (IoT) field has accelerated, and various devices have become wireless. In response to this, antennas are required to be not only small and thin, but also flexible enough to be installed. Artificial Magnetic Conductor (AMC) is a metamaterial which exhibits a Perfect Magnetic Conductor (PMC) characteristics with a reflection phase of 0 degrees within a certain frequency [1]. Using the AMC has advantages such as maintaining the antenna characteristics on the metal, improving the gain, reducing the back lobe radiation, and miniaturizing the antenna size $[2,3]$. Therefore, AMC is used in the microwave device as the ground plane, the reflector, or the Electro-Magnetics (EM) wave absorber. In these research backgrounds, there is a demand for further miniaturization and thinning of the substrate. An AMC substrate with a circular and Jerusalem shape achieving the decrement of overall size is presented to reduce the size [4], and an AMC structure with slot is also presented [5]. We investigate an AMC substrate composed of the square patch and the semicircle notch to reduce the thickness of the AMC substrate.

\section{Analysis model}

Figure 1(a) shows the analysis model of the AMC unit cell. The AMC consists of the ground plane, the dielectric layer with thickness of $h$, and the metal patch. Figure 1(b) shows the boundary condition of the unit cell structure. To simulate the reflection phase with a periodic boundary condition, each of the facing walls are set such that they are identical to those of a PEC and a PMC. The plane wave is excited from the top of the unit cell structure. Figure 1(c) shows the analysis model of the square $2 \times 2$ patch AMC with a semicircle notch. In the analysis model of the AMC, the boundary condition near the semicircular notch is complicated. For this reason, we use the analysis model of $2 \times 2$ patch AMC. Figure $1(\mathrm{~d})$ shows the design parameters of the AMC, where $\lambda_{0}$ is the wavelength corresponding to $f_{0}$ at the frequency of the reflection phase of 0 degrees. FR-4 (relative permittivity: $\varepsilon_{r}=4.2$, dielectric loss tangent: $\tan \delta=0.025$, thickness of the conductor: $t=18 \mu \mathrm{m}$ ) is used for the dielectric substrate. The characteristics of the proposed AMC are analyzed and compared with those of the square patch AMC, by using the full wave simulation with Computer Simulation Technology Microwave Studio 2020 (CST MWS 2020) [6].

\section{Analysis result}

Figure 1(e) shows the analyzed reflection phase characteristics of the proposed AMC when the arc length $C / W$ is changed. Here, $C / W$ is the arc length of the semicircle normalized by the width $W$. The horizontal axis is the frequency $f$ normalized by $f_{0}$. The vertical axis is the reflection phase of the AMC. It is found that the frequency with the reflection phase of 0 degrees is shifted to the lower side. Furthermore, it is found that the second frequency with the reflection phase of 0 degrees appears. Figure 1(f) shows the analyzed $S_{11}$ characteristics. Where, solid line is the analyzed $S_{11}$ characteristics of dielectric loss tangent: $\tan \delta=0.025$ (FR-4), dashed line is that of $\tan \delta=0$ (no loss). For $\tan \delta=0.025$ in Fig. 1(f), it is found that the frequency 


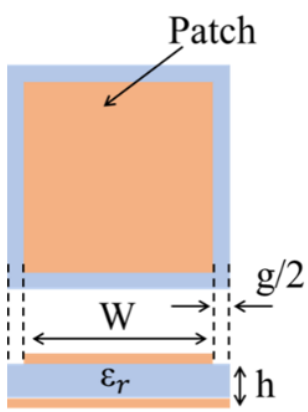

(a) Analysis model of AMC unit cell.

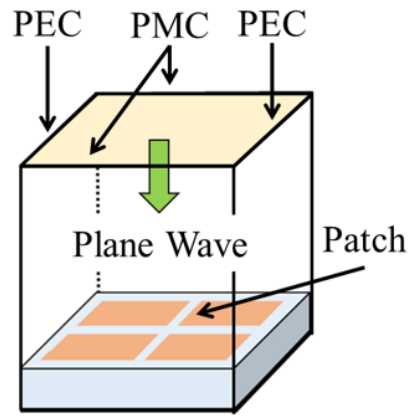

(b) Boundary condition.

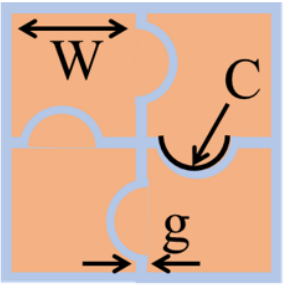

(c) Square $2 \times 2$ patch with semicircle.

(d) Parameters of AMC.

\begin{tabular}{cccc}
\hline $\begin{array}{c}\text { Operation } \\
\text { frequency } \\
f_{0}[\mathrm{GHz}]\end{array}$ & $\begin{array}{c}\text { Width } \\
W[\mathrm{~mm}]\end{array}$ & $\begin{array}{c}\text { Gap } \\
g[\mathrm{~mm}]\end{array}$ & $\begin{array}{c}\text { Thickness } \\
h[\mathrm{~mm}]\end{array}$ \\
\hline 11.0 & $0.20 \lambda_{0}$ & $0.037 \lambda_{0}$ & $0.0293 \lambda_{0}$ \\
\hline
\end{tabular}

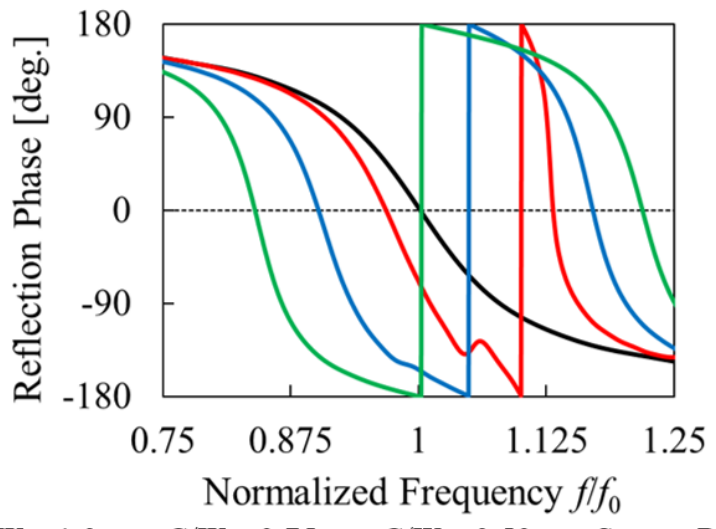

$-C / W=1.0-C / W=0.75-C / W=0.50-$ Square Patch

(e) Analyzed reflection phase characteristics.

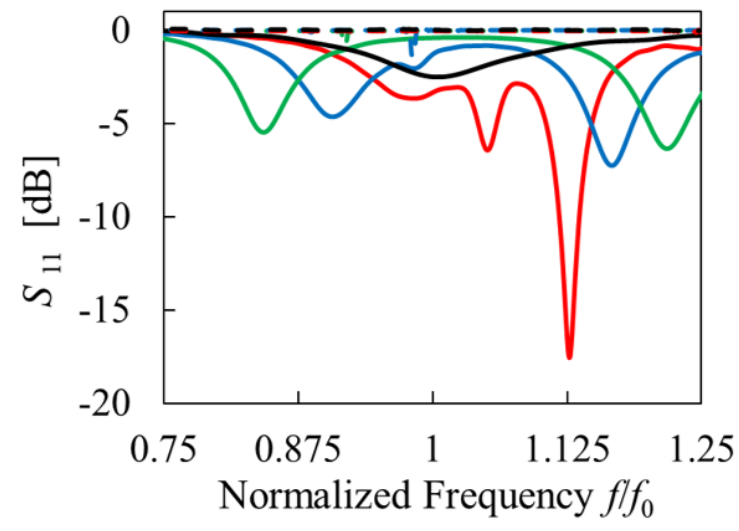

Green: $C / W=1.0$ Blue: $C / W=0.75$ Red: $C / W=0.50$ Black: Square Patch

Solid line: $\tan \delta=0.025$ (FR-4) Dashed line: $\tan \delta=0$ (no loss)

(f)Analyzed $S_{11}$ characteristics.

Fig. 1. The structure and analysis result.

with the reflection phase of 0 degrees is shifted to the lower side, while the reflection loss increases. However, there is no significant difference between the respective $S_{11}$ characteristics. For $\tan \delta=0$ (no loss) in Fig. 1(f), the $S_{11}$ characteristics is 
about $0 \mathrm{~dB}$ and reflects without loss. Therefore, the $S_{11}$ characteristics is considered to be due to the dielectric loss. Table I shows the normalized frequency and the AMC substrate thickness of electrical length. From Table I, it is found that the AMC substrate thickness is thinning about $15 \%$ compared with the square patch AMC that has no semicircle notch. Therefore, it is found that the frequency with the reflection phase of 0 degrees is shifted to the lower side by increasing the arc length $C / W$, and the AMC substrate thickness is thinning about $15 \%$ compared with the normal square AMC.

Table I. Simulation result of the proposed AMC.

\begin{tabular}{ccc}
\hline $\begin{array}{c}\text { Arc length } \\
C / W\end{array}$ & $\begin{array}{c}\text { Normalized frequency } \\
f / f_{0}\end{array}$ & $\begin{array}{c}\text { Thickness } \\
h[\mathrm{~mm}]\end{array}$ \\
\hline 0.50 & 0.967 & $0.0284 \lambda$ \\
0.75 & 0.900 & $0.0264 \lambda$ \\
1.0 & 0.839 & $0.0246 \lambda$ \\
\hline
\end{tabular}

\section{Measurement method}

We experimentally investigate the reflection phase characteristics of the proposed AMC substrate as shown in Fig. 1(c) when the arc length $C / W$ is changed in three ways: $0.5,0.75$, and 1.0 as shown in Table I. In the experiment, transmit and receive electromagnetic waves from one horn antenna and measure the reflection phase of each of the AMC substrate and the metal plate for the reference. To remove unwanted reflections, the gating function of time-domain kit of the vector network analyzer is used. The reflection phase of the AMC substrate is then derived using Eq. (1).

$$
\text { Reflection phase }: \theta_{\mathrm{AMC}}-\theta_{\mathrm{PEC}}+\pi
$$

Where, $\theta_{\mathrm{AMC}}$ is the measured reflection phase for the AMC substrate, $\theta_{\mathrm{PEC}}$ is that of the metal plate, and $\pi$ is the ideal reflection phase of the metal plate. Note that the measurement of the reflection phase when a plane wave is irradiated is pseudo-feasible because the sample is placed in close contact with the aperture of the horn antenna and is not affected by external unwanted reflections. FR-4 (relative permittivity: $\varepsilon_{r}=4.2$, dielectric loss tangent: $\tan \delta=0.025$, thickness of the conductor: $t=18 \mu \mathrm{m}$ ) is used for the dielectric substrate.

\section{Measurement result}

Figure 2(a) shows the prototype AMC substrate and its dimensions of this AMC substrate are $153.6 \mathrm{~mm} \times 192 \mathrm{~mm}$ to cover the aperture of the horn antenna. Figure 2(b) shows the measurement result of the reflection phase of the proposed AMC when the arc length $C / W$ is changed. The horizontal axis is the frequency $f$ normalized by $f_{0}$, and the vertical axis is the reflection phase. From Fig. 2(b), it is found that the frequency at the frequency of the reflection phase of 0 degrees shifts to the lower side by increasing the arc length $C / W$. Compared to the square patch AMC substrate without the semicircular notch, the proposed AMC substrate with the semicircular notch is thinning by changing the arc length $C / W$. 



(a) Prototype AMC substrate.
$-C / W=1.0-C / W=0.75-C / W=0.50-$ Square Patch

(b) Measured reflection phase characteristics.

Fig. 2. Measured reflection phase characteristics and prototype AMC substrate.

\section{Conclusion}

In this paper, we investigated the AMC substrate applying the square patch with the semicircle for substrate thinning. It was confirmed that the proposed AMC substrate is thinning about $15 \%$ compared with the normal square patch AMC by increasing the arc length of the semicircle of the proposed AMC. Furthermore, measurements were taken and we also experimentally confirmed the proposed AMC characteristics in measurement. 